\title{
Mucosal Manifestation of Lupus Erythematosus
}

\section{Dr. Thiago de Almeida Prado Naves Carneiro, DDS; MSc \& PhD}

Post-Doctoral Research fellow, Department of Occlusion, Fixed Prostheses, and Dental Materials, School of Dentistry, Universidade Federal de Uberlândia, Uberlândia, Minas Gerais Brazil

\section{Dr. Livia Bonjardim Lima, DDS; MSc}

PhD Student, Department of Occlusion, Fixed Prostheses, and Dental Materials, School of Dentistry, Universidade Federal de Uberlândia, Uberlândia, Minas Gerais Brazil

\section{Dr. João Paulo Silva Servato, DDS; MSc \& PhD}

Professor, School of Dentistry, University of Uberaba (UNIUBE), Uberaba, Minas Gerais, Brazil

\section{Dr. Luiz Fernando Barbosa de Paulo, DDS;MSc \& PhD}

Program of Specific Care of Oral Diseases, Stomatology Diagnosis Unit, HC, Federal University of Uberlândia, Uberlândia, Minas Gerais, Brazil

\begin{abstract}
Lupus Erythematosus is an autoimmune rheumatic disease characterized by the action of antibodies against a variety of antigens. A large spectrum of oral mucosal lesions can be found in the systemic and cutaneous forms of lupus erythematosus. The present article reports a 67-year-old woman with Lupus Erythematosus associated with oral mucosal lesions.
\end{abstract}

Keywords: lupus erythematosus; oral lesions; autoimmune disease.

\section{Case Report}

Lupus Erythematosus is a classic example of autoimmune rheumatic disease, characterized by the action of antibodies against a variety of auto antigens. Biett in1828 and Kaposi in 1872first described lupus erythematosus. The presentation of this autoimmune rheumatic diseasecan vary from a simple skin rash to progressive multiorgan disease. A large spectrum of oral mucosal lesions can be found in the systemic and cutaneous forms of lupus erythematosus ${ }^{1}$.Typical cases of oral lesions are clinically characterized by the presence of white papules, central erythema, a border zone of irradiating white striae and peripheral telengiectasia ${ }^{2,3,4}$. In this way, the objective of the present article is to present a case of Lupus Erythematosus associated with oral mucosal lesions.

A 67-year-old woman presented with burning mouth symptomatology for several months was reported to our Private Clinic. Past medical history reveals chronic cutaneous lupus erythematosus and hypertension. Extra oral examination, obviously pointed to skin lesions on the scalp which appeared as depigmented scarring alopecia (Panel A). She also had a hyperkeratotic papules in elbows (Panel B). Intra- oral avaliation shows multiples erythematous lesions with difuse reddish marginal areas, and central areas with epithelium desquamation (Panel C, D e E). Generalized bleeding on probing was present. There were no periodontal pockets, furcation involvement, and mobility. Excisional biopsy was done under local anesthesia in the maxillary anterior region. Specimen was sent for histopathologic examination and confirms the clinical diagnosis. The patient was treated with systemic corticosteroids and total resolution of mucosal manifestations was achieved in 2 months. After one year follow up no recurrence was found. 

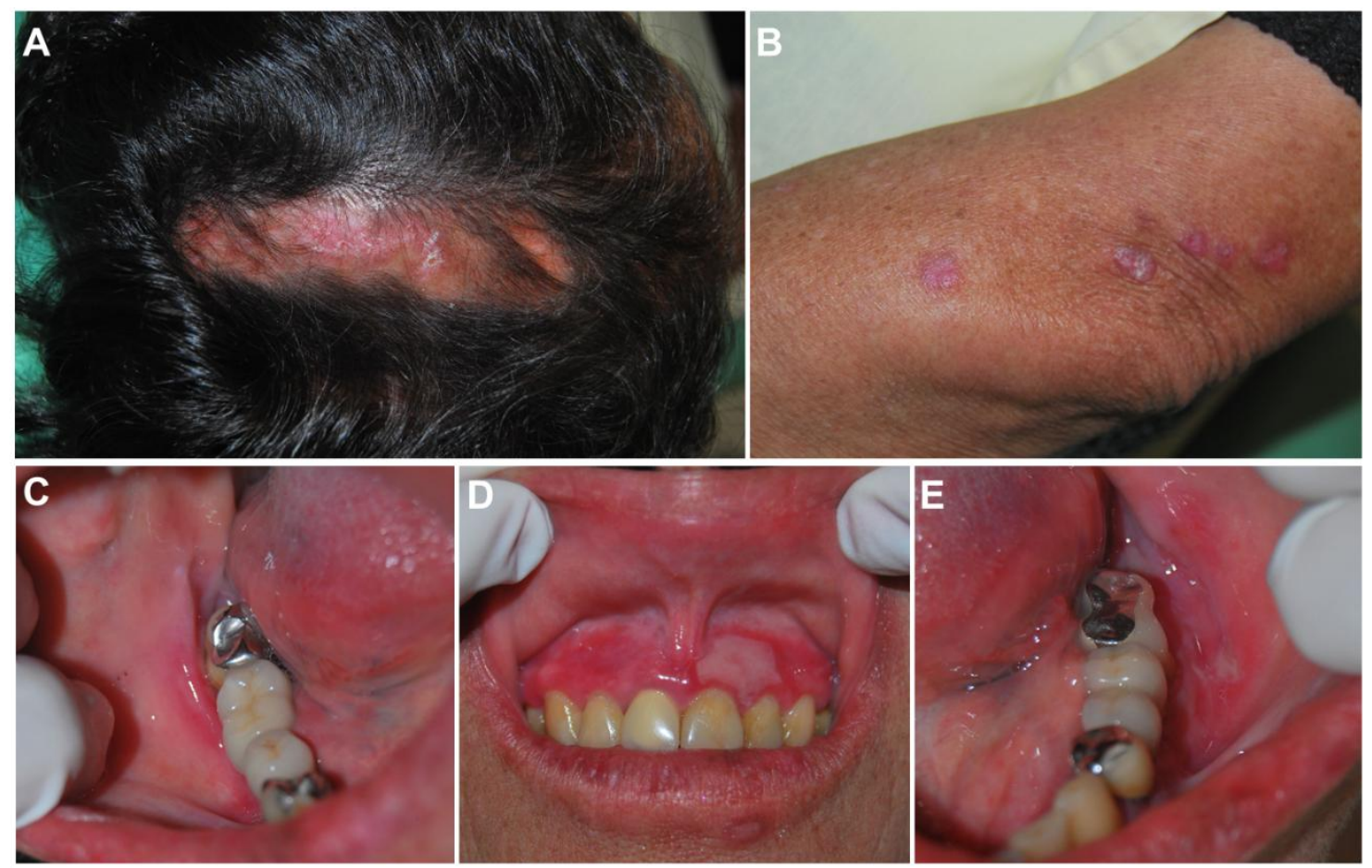

Figure 1. A - Skin lesions on the scalp which appeared as depigmented scarring alopecia. B - Presence of hyperkeratotic papules in elbows. $C, D, E$ - multiples erythematous lesions with difuse reddish marginal areas; and central areas with epithelium desquamation.

\section{REFERENCES}

[1] Ramakrishna Y, Reddy JS. Systemic lupus erythematosus presenting with oral

[2] mucosal lesions--a case report. J ClinPediatr Dent. 2009 Spring;33(3):255-8.

[3] SchiØdt M, Pindborg JJ. Oral discoid lupus erythematosus- The validity of previous histopathologic diagnostic criteria. Oral Surg. 1984;57:46-51.

[4] Neville BW, Damn DD, Allen CM, Bouquot JE. 2nd ed. Philadelphia: Elsevier; 2005. Oral and maxillofacial pathology.

[5] Gisslen H, Heyden G. Histological and histochemical investigations of lupus erythematosus in the skin and the oral mucosa. ActaDermVenereol. 1975; 55: 57-63. 